\title{
Hallazgos sobre la alteración de la señal auditiva aferente cortical en la enfermedad de Ménière
}

\author{
Findings on the alteration of the cortical afferent auditive signal \\ in the Ménière disease
}

\author{
Ried U. Ernesto1; Ried G. Ernesto1; Martínez Cristián².
}

\begin{abstract}
RESUMEN
Se presenta un trabajo retrospectivo, donde se analizaron las respuestas del sistema auditivo aferente central en 36 enfermos que presentaban vértigos y desequilibrio, hipoacusia fluctuante y tinnitus que clínicamente fueron considerados portadores de la Enfermedad de Ménière clásica.

Se estudiaron con los exámenes otoneurológicos habituales, y además se estudió la vía auditiva aferente central con el mapeo cerebral auditivo promediado. Este estudio se realizó en todos estos pacientes mediante la estimulación sonora de un oído, y posteriormente se registraron las respuestas aferentes en la corteza cerebral hasta los 15 milisegundos, a través de un electroencefalógrafo computarizado de 22 canales, y promediándolas para eliminar actividad cerebral no auditiva, lo que permitió a través de la Transformada rápida de Fourier, hacer un mapeo topográfico de las respuestas eléctricas del tronco cerebral y de las zonas cerebrales que procesan la audición. Luego de las respuestas de un oído se examinó sucesivamente el otro oído.

Los mapeos cerebrales así obtenidos en los 36 enfermos portadores la Enfermedad de Ménière unilateral, y repetidos en fases de hipoacusia, como de normalidad auditiva, se compararon con los que se obtienen en las personas sanas y sin patología auditiva.

$\theta$ hallazgo más relevante fue: en todos los enfermos de Ménière encontramos alteración de la señal aferente a nivel de la corteza cerebral auditiva primaria contralateral y de la corteza auditiva secundaria ipsilateral.

Esta respuesta de la corteza cerebral auditiva, diferente de la que encontramos en las personas normales, podría ser útil para buscar el origen de la Enfermedad de Ménière.

Palabras clave: Enfermedad de Ménière; vía aferente auditiva; Mapeo Cerebral Auditivo.
\end{abstract}

\section{SUMMARY}

A retrospective paper is presented, where the responses of the central afferent auditive system in 36 patients suffering from vertigo and imbalance, fluctuating

\footnotetext{
${ }^{1}$ Médico Otorrinolaringólogo Instituto de Otología, Santiago de Chile.

2 Tecnólogo Médico Instituto de Otología, Santiago de Chile.
} 
hypoacusia and tinnitus who were clinically considered bearers of the classical Ménière Disease were checked.

They were checked with the normal otoneurological exams and the central afferent auditive conduct was studied with the auditory cerebral mapping averaged. This study was made on all these patients through the sound stimulation of one ear, and

later the afferent responses of the cerebral cortex were recorded up to 15 milliseconds, by means of a 22 channel computerized electroencephalograph, and averaging them to eliminate non auditive cerebral activity, which allowed to make a topographic mapping of the electrical responses of the cerebral cortex and of the cerebral zones that process audition through the Fourier quick Transformer.

After the responses of one ear, the other ear is successively examined.

The cerebral mappings obtained from the 36 patients with unilateral Meniere Disease, and repeated in hypoacousia stage, as well as auditive normality, were compared to those obtained from healthy persons without auditive pathology.

The most relevant finding was: in all Ménière patients we found alterations of the afferent signal at the level of the contralateral primary auditive cerebral cortex and of the secondary ipsilateral auditive cortex.

This response from the auditive cerebral cortex, different to what we find in normal people, could be useful to find the origin of the Ménière Disease.

Key words: Meniere Disease; auditive afferent channel; Cerebral Auditive Mapping.

\section{INTRODUCCIÓN}

La etiología de la enfermedad de Ménière persiste desconocida hasta ahora.

Sin embargo, sí se conoce su manifestación clínica que, según el Comité de Audición y Equilibrio de la Academia Americana de Aorrinolaringología y Grugía de Cabeza y Ouello -en 1972 y 1995- se caracteriza por un conjunto de síntomas: crisis de desequilibrio, con o sin sensación de vértigo rotatorio; hipoacusia sensorioneural fluctuante (con mejoría y empeoramiento de la capacidad auditiva ocasionales), que en un comienzo afecta las fre cuencias graves; tinnitus; y a veces una sensación de plenitud de la cabeza o del mismo oído.

Se ha postulado, desde la época de Prosper Ménière (1861), como una enfermedad de la cóclea, o puramente periférica'.

Histológicamente se ha encontrado que hay dilatación de la escala media de la cóclea, y a veces del sáculo (Hallpike 1938), pero esta dilatación es variable y a veces no se le encuentra.

Se han planteado múltiples teorías para explicar estos hechos, algunas de ellas basadas en la hipótesis de la perturbación del flujo longitudinal de endolinfa desde la estría vascular hasta el saco endolinfático, o por mala función del saco².

Sin embargo, actualmente nuevas teorías como William Gibson ${ }^{3}$, sostienen que el flujo sería radial. Este comenzaría en la estría vascular hacia la escala mediay de allí fluiría a la escala timpánica y vestibular, y se reabsorbería de nuevo en la estría vascular. Por otra parte, las mediciones de las presiones en las escalas de la cóclea, demuestran que la dilatación de la escala media ocurre en ausencia de hipertensión en ella (lo que se ha observado en animales con obliteración del saco endolinfático).

Por lo tanto, y después de descartar afecciones centrales (como neuromas, tumores y afecciones vasculares centrales, etc.) y patologías periféricas (como laberintitis, fístulas, otoesclerosis, dehicencias del canal semicircular externo, traumas etc.) se puede llegar al diagnóstico de lo que se llama Enfermedad de Ménière clásica, frecuentemente con hídrops endolinfático, pero cuya etiopatogenia persiste desconocida.

Se ha pensado que pudiera ser una enfermedad autoinmune del oído interno, (Mac Cabe, 1979, 
Hughes, 1988), pero los estudios posteriores no son concluyentes, (Rauch et al., 2000).

También se ha pensado en factores alérgicos como etiología del Ménière, (Derebery et al., 1999), y se han postulado múltiples tratamientos, desde antihistamínicos, esteroides locales y generales, inmunoterapias... pero tampoco han sido de eficacia concluyente.

Aros estudios sugieren que las aferencias del sistema propioceptivo, particularmente la muscula tura y articulaciones cervicales, serían gatillantes de las crisis de vértigo ${ }^{4-5}$. Se ha relacionado también con equivalentes de migraña, con aura y síntomas vestibulares, con o sin cefalea ${ }^{6}$, y se postula que sería causado por disfunción focal cerebral y del tronco cerebral. A este respecto, Lance propone que una desinhibición excesiva de la aferencia sensitiva del trigémino (afección en la cara, senos o incluso por enfriamiento brusco del maxilar, como por ejemplo tomar helados o hielo), podría desencade nar una migraña con mareo, por alteración del procesamiento de las señales sensoriales a nivel cerebral ${ }^{7}$. Woodhouse y Drummond, encontraron que la fonofobia, la algiacusia y el disconfort por sonidos intensos sería un fenómeno de sobrecarga sensorial central ${ }^{8}$. Lo mismo se ha demostrado por Podoshin, quien demostró que la latencia de la onda 5 de la audiometría de respuestas evocadas del tronco cerebral (ABR o $\mathrm{EAA}$ ), estaba alargada en los pacientes con migraña y mareo ${ }^{9}$.

También Penfield ha comprobado que la estimulación eléctrica de la zona del Girus Silviano superior, produce sensación subjetiva de movimiento ${ }^{10}$.

La explicación fisiopatológica de cómo una disfunción auditiva y vestibular central pueda originar una sordera unilateral fluctuante y con debilidad en la respuesta vestibular a la estimulación calórica, (que pareciera ser periférica) sería de dos tipos:

- Una, dada por Ishiyama, demostró en chinchillas que las señales aferentes del neuroepitelio vestibular periférico son moduladas por fibras eferentes originadas en el cerebro ${ }^{11}$ y que hacen sinapsis en la base de las células ciliadas vestibulares, actuando a través de los neurotransmisores Acetilcolina y neuropéptidos de Calcitonina produciendo inhibición o facilitación de las respuestas aferentes. Figura 1.

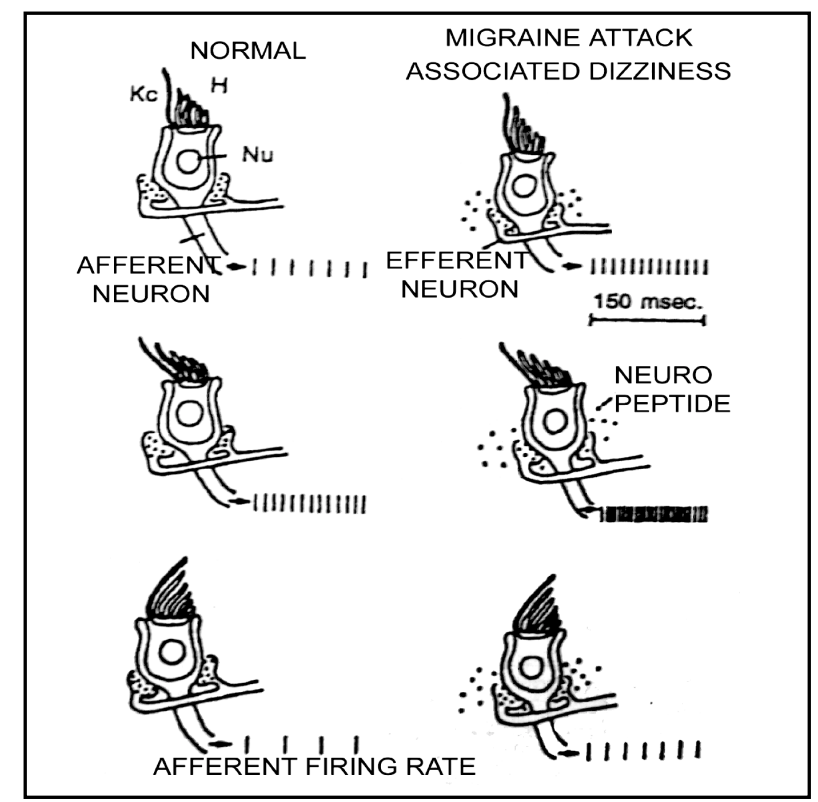

Figura 1. Mecanismo de modulación de la aferencia vestibular causado por la liberación de neurotransmisor neuropéptido de calcitonina en las terminaciones eferentes vestibulares periféricas. B y $\mathrm{C}$ muestran que al doblarse la esterocilia hacia o desde el kinocilio, aumenta o disminuye la frecuencia de descarga aferente vestibular (Según Outrer y Baloh). 
- La otra explicación, dada por Shuerger, demuestra por histoquímica la existencia de modificación de las señales aferentes en los núcleos vestibulares por señales eferentes, provenientes del núcleo Locus Cerúleo noradrénergico ${ }^{12}$.

Outrer y Baloh postulan que la inhibición de origen central de la sensibilidad vestibular, a través de las vías eferentes, sería responsable de la intolerancia a los movimientos, si es que los dos lados están afectados simétricamente, en cambio si un solo lado está afectado se produciría vértigo espontáneo. Esta modulación de la sensibilidad vestibular estaría mediada por neurotransmisores que se liberan en las terminaciones eferentes ${ }^{13}$.

日 Dr. Jonhson ha asociado también los mareos y vértigos con cuadros de depresión, ansiedad, ataques de pánico, y ha indicado tratamientos para los mareos, en base a medicamentos elevadores de los niveles de neurotransmisores en las sinapsis $^{14-15}$.

Nosotros publicamos en 1993 la hipótesis, (basada en observaciones clínicas) de que el estrés, la tensión o la depresión podría producir "Sordera por inhibición retrococlear eferente" ${ }^{16}$.

Según Kim y colaboradores, que efectuaron una numerosa encuesta entre otoneurólogos y otólogos (2005), sobre el diagnóstico y el tratamiento de la enfermedad de Ménière, aún no existe consenso sobre ellos ${ }^{17}$. En nuestro medio se ha diseñado un examen para facilitar el diagnóstico ${ }^{18}$, y se le otorga cada vez más importancia a la influencia de la vía eferente sobre la aferente auditiva y vestibular ${ }^{19}$.

Además, desde hace muchos años, se ha ido observando una relación entre la Enfermedad de Ménière y los trastornos del ánimo y el estrés, junto con sinusitis (Otoneuropsiquiatría del Dr. A. Tello, Chile) ${ }^{20}$.

Tello postulaba, que un "estímulo inadecuado interno" (sinusitis) más un "estímulo inadecuado externo" (el estrés), serían los desencadenantes de la Enfermedad de Ménière.

Además se ha observado que aparece junto a personalidades muy responsables, perfeccionistas y muy estructuradas ${ }^{15}$.

Para intentar entender si la enfermedad de
Ménière es sólo una enfermedad periférica-coclear, o si su etiopatogenia podría ser o tener un componente central, es que se decidió revisar los exámenes de mapeo cerebral auditivo promediado en pacientes portadores de Enfermedad de Ménière unilateral, tanto en estado de crisis con hipoacusia sensorioneural, asi como en estado entre crisis y normalización auditiva. Luego, los resultados se compararon con los de personas normales, sanos y sin patología auditiva, principalmente a nivel de la corteza auditiva primaria contralateral y la corteza auditiva secundaria ipsilateral.

\section{MATERIAL Y MÉTODO}

Se revisaron retrospectivamente las fichas de 36 pacientes adultos que, de acuerdo a la historia clínica y exámenes otoneurológicos, fueron considerados portadores de la Enfermedad de Ménière clásica unilateral. Tenían entre 27 a 76 años de edad, de los cuales 17 eran de sexo masculino y 19 de sexo femenino. Todos ellos fueron atendidos en el Instituto de Otología de Santiago de Chile entre enero de 1998 y diciembre de 2004.

En todos ellos se había descartado lesiones orgánicas centrales por tomografia axial computada o resonancia nuclear de cerebro y oídos.

Todos ellos fueron estudiados con los exáme nes otoneurológicos habituales y además con el Mapeo Cerebral Auditivo Promediado21, que consiste en evaluar la vía auditiva aferente central, hasta los 15 milisegundos, después de aplicar un estímulo sonoro de $80 \mathrm{~dB} \mathrm{H}$, con un fono en un oído, proveniente de un generador de tonos clicks. Se repitió con una frecuencia de 8 clicks por segundo, hasta completar 4000 estímulos, con la finalidad de obtener una buena promediación de las respuestas cerebrales.

Pasados los $15 \mathrm{~ms}$ ya no se pudieron obtener respuestas promediables, y las que se obtuvieron en latencias medias y largas no fue posible interpretarlas.

Los registros se obtuvieron mediantela colocación de electrodos a nivel del cuero cabelludo, en forma similar a un electroencefalograma de 22 canales. 
Esta señal recogida en cada electrodo es amplificada en forma independiente y filtrada para tonos agudos y graves. La señal análoga fue convertida en digital mediante un oscilador y fue registrada con un electroencefalógrafo computarizado Akonic Bio-PC, (Colombi Buenos Aires Argentina).

Mediante la aplicación de la Transformada rápida de Fourier, se interpoló la señal de los distintos electrodos formando mapas de la actividad eléctrica cerebral.

日 patrón "normal" de respuestas promediadas auditivas evocadas, y visualizadas en corteza auditiva por mapeo cerebral, fue descrito en un trabajo previo 20 , por los mismos autores, destacando el registro de respuestas del colículo inferior a los $5.5 \mathrm{~ms}$, el del geniculado medial a los $6.5 \mathrm{~ms}$ y luego las respuestas de la corteza cerebral auditiva primaria contralateral a los $7.6 \mathrm{~ms}$, y de la corteza auditiva ipsilateral a los $8.9 \mathrm{~ms}$.
Todos los registros se efectuaron bajo condiciones controladas, en silencio y con los enfermos sentados confortablemente. Para cada examen, se estimuló un oído y se registraron las respuestas cerebrales, y luego se estimuló el otro oído y se registran sus respuestas separadas del primer oído, luego se hicieron 10 mapas topográficos en colores, de las respuestas cerebrales evocadas por la estimulación de cada oído.

\section{RESULTADOS}

A analizar los registros obtenido con el "Mapeo Cerebral Auditivo Promediado", en los pacientes portadores de Enfermedad de Ménière, se encontró que las respuestas en los enfermos de Ménière se encontraban ausentes o alteradas a nivel de la corteza auditiva primaria contralateral al oído esti-

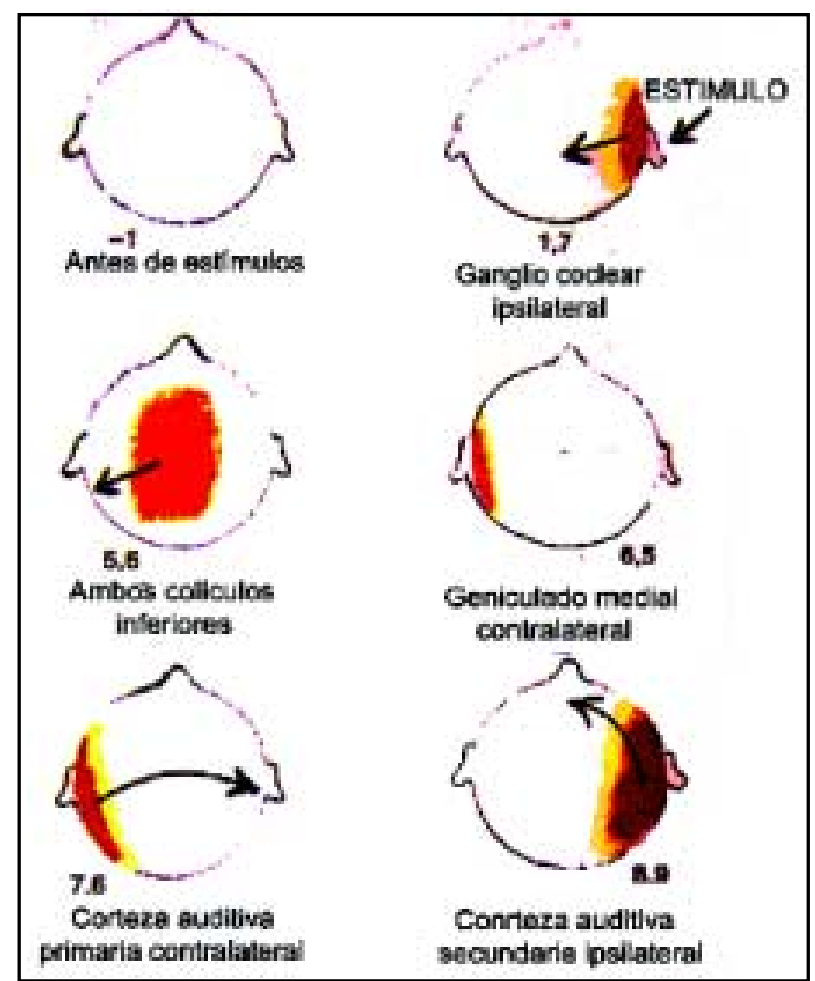

Figura2. Patrón de respuestas del mapeo electroencefalográfico auditivo promediado obtenido en sujetos normales al estimular oído derecho. Latencias en milisegundos. 
mulado, y de la corteza auditiva secundaria ipsilateral al oído estimulado (respuestas obtenidas entre los 6 y $9 \mathrm{~ms}$ ), tanto en fase de hipoacusia, como en las fases de normalización auditiva.

Estas respuestas alteradas de las cortezas auditivas fueron similares, cuando se estimulaba el oído sano o el que había estado hipoacúsico.

A comparar estos mapeos con los obtenidos de sujetos sanos, se pudo observar que las respuestas son diferentes a lo que habíamos detectado como patrón normal. Figura 2.

Aro hallazgo interesante es, que muchos de estos enfermos de Ménière portadores de audiometría con hipoacusia sensorioneural para frecuencias graves presentaban respuestas casi normales para la onda 1,3 y 5 del examen de respuestas evocadas de tronco cerebral ( $\boxminus R A$ o BERA) , y alteradas en la corteza auditiva.

Con este examen de Mapeo Cerebral Auditivo Promediado efectuado a los enfermos de Ménière encontramos que: que al estimular el oído hipoacúsico, hay respuesta casi normal en núcleo coclear ipsilateral y en ambos colículos inferiores,y luego muy débil respuesta en intensidad del geniculado medial contralateral y enseguida hay ausencia de respuesta de la corteza auditiva primaria contralateral y veces una débil respuesta de la corteza auditiva secundaria ipsilateral, así como de las áreas de asociación frontales y occipitales, (que responden entre los 10 y $15 \mathrm{~ms}$ ) Figura 3, sin observar aumento de las latencias.

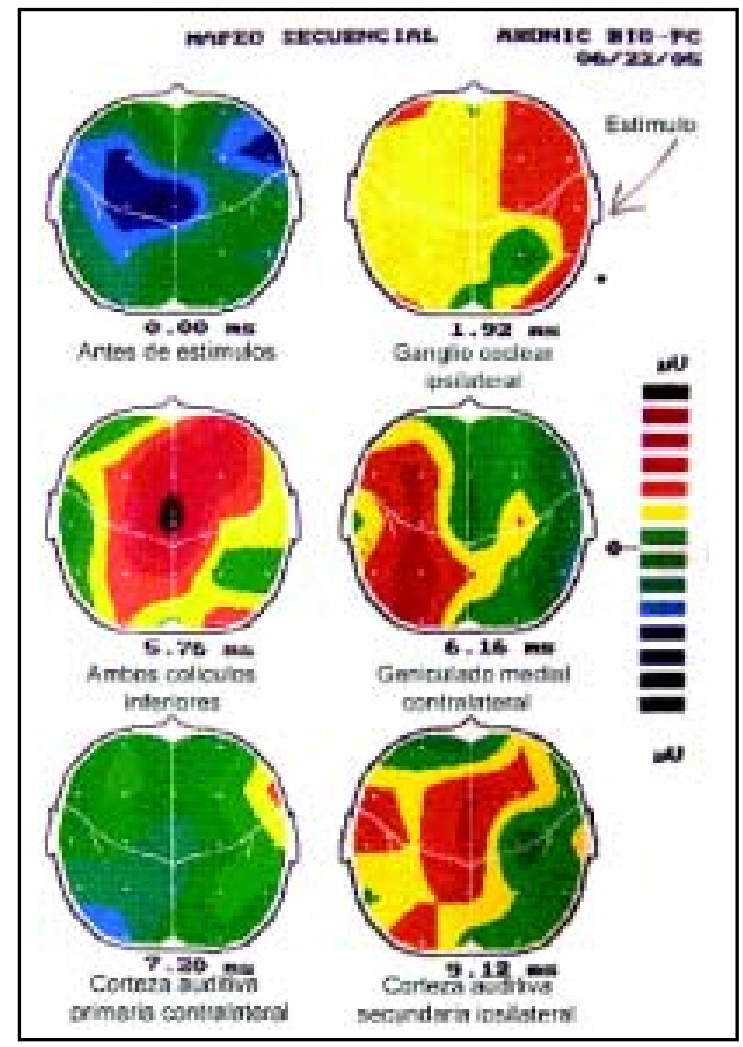

Figura 3. Respuestas obtenidas en un enfermo de Méniere al estimular oído derecho. Latencias en milisegundos. Se puede apreciar que a los $7.20 \mathrm{~ms}$ no hay respuesta en corteza auditiva primaria contralateral y a los $9.48 \mathrm{~ms}$ no hay tampoco respuesta en la corteza auditiva secundaria ipsilateral, sino que en otras zonas del cerebro. 


\section{DISCUSIÓN}

Los resultados de los mapeos cerebrales en pacientes portadores de enfermedad de Ménière, al ser comparados con los de sujetos normales, dejan en evidencia que los enfermos de Ménière presentan un patrón de respuestas en la corteza cerebral auditiva, diferente al esperado como normal, tanto al estimular el oído sano, como el hipoacúsico, aún en períodos en que la fluctuación auditiva normaliza la audición.

La presencia de ERA o BERA (potenciales evocados de tronco cerebral) con tiempos de conducción normal hasta la onda 5 , cuado se estimula a $85 \mathrm{~dB} \mathrm{HL}$, en períodos de hipoacusia sensorioneural, hace pensar que la alteración podría corresponder a un fenómeno central que tiene manifestaciones a nivel periférico.

Hay que considerar además que las vías auditivas y vestibulares eferentes, se originan en la misma zona de la corteza cerebral, que hemos detectado que en los enfermos de Ménière funciona diferente al patrón aferente normal.

Por lo tanto podríamos deducir que esta "disfunción" central podría producir una disminución o bien un aumento de la función moduladora eferente, determinando una disfunción del sistema cócleo vestibular, por medio de la alteración de las vías, los núcleos, y los órganos periféricos auditivo y vestibular.

Podríamos también pensar que los enfermos de Ménière tienen una falla en los niveles de neurotransmisores a nivel de las sinapsis, entre vías aferentes y eferentes, y ello causaría sus síntomas, y por eso también los medicamentos Ilamados antidepresivos, que son moduladores de los niveles de neurotransmisores en las sinapsis de todo el sistema nervioso central, podrían ser útiles, para el tratamiento del Ménière, y posiblemente algunos tinnitus y sorderas súbitas, que posible mente tengan el mismo origen.

Oreemos que falta desarrollar estudios en otras afecciones cocleares para entender mejor e interpretar adecuadamente nuestros resultados.

\section{BIBLIOGRAFÍA}

1. ATKINSON M. Ménière's original papers: reprinted with an English translation together with commentaries and biographical sketch. Acta Otolaryngol 1961; (suppl 162): 1-78.

2. PAPARELA MM. Pathogenesis and pathophisiology of Ménière Disease. Acta Otolaryngol (Stockh) 1991;485 (suppl):26-35.

3. WILLIAM GiBson. Comunicación personal. 2002.

4. WRISLEY, DM., ET AL. Cervicogenic dizziness: a review of diagnosis and treatment. $J$ Orthop Sports Phys Ther 2000; 30(12): 75566.

5. DeEUCaH E Comunicación personal. 1978.

6. JOHNSON D. G_enN. Medical Management of Migraine related Dizziness and Vertigo. Laryngoscope 108: January 1998 Supplement.

7. LANCE JW. Ourrent concepts of migraine pathogenesis. Neurology 1993; 43: S 11-5.

8. WOODHOUSE A, DRUMMOND PD. Mechanism of increased sensitivity to noise and light in migraine headache. Cephalalgia 1993; 13: 41721.

9. Podoshin, L Ben David J, Pratt H et al. Auditory Brainstem evoked potential in patient with migraine. Headache 1987; 27: 27-9.

10. PENFEnd W. Vestibular sensation and the vestibular cortex. Ann Otol 1957; 66: 691-8.

11. ISHYYAMA A, LÓPEZ I, WACKYM PA. Subcelular innervation patterns of the calcitonin generelated peptidergic efferent terminals in the chinchilla vestibular periphery. Otolaryngol Head Neck Surg 1994; 111: 385-95.

12. SCHURGR RJ, BALABAN CD. Immunohistochemical demonstration of regionally selective projections from locus coeruleus to the vestibular nuclei in rats. Exp Brain Res 1993; 92: 351-9.

13. OIRG RM, BALOH RW. Headache 1992; 32: 300-4.

14. Gover V, JARMAN J, SANDLER M. Migraine and depression: Biological aspects. J Psychiatr Res 1993; 27: 223-31.

15. DI DURO, J. Vestibular Migraine: objective diagnostic criteria . Neurol Sci 24 2003; 80-1.

16. Riø U. E, Riø G E, Peragallog A, Adrian H. Síndrome de sordera por inhibición retroco- 
clear eferente. Acta Otorrinolaringológica Española 1993; 44: 253-5.

17. Kim H. Harold, Wiet J.Riahard, Battista A. RoBERT. Trends in the diagnosis and the management of Meniere disease: Result of a survey. Oolaryngology - Head and Neck Surgery 2005; 132: 722-6.

18. Riveros M. Héctor, Cohen V. Mauricio, Badía V. Pedro, anabalón B. José luis, correa G QAUDIO, Utilidad de la prueba calórica mínima. Rev Atorrinolaringol Ar Cabeza Ouello 2005; 65: 193-6.
19. Déano Paul, Robles Ignacio, Robles luis. Sistema eferente auditivo. Rev Otorrinolaringol Or Cabeza Quello 2005; 65: 55-62.

20. Telo ARTuRo. Otoneuropsiquiatría, Santiago, 1971; Editorial Andrés Bello.

21. Ri甲d U. ERnesto, Ri巴d G ERnesto, martínez CRISTIAN. Nuevo método para el estudio clínico de la vía auditiva, en tronco cerebral y en corteza auditiva primaria y secundaria, utilizando el mapeo cerebral auditivo promediado en los prime ros 15 ms después de aplicar sonido en un oído. Acta Otorrinolaring Esp 1999;0 50(5): 349-353.

Dirección: Dr. Ernesto Ried Undurraga Instituto de Otología

Calle: Felix de Amesti 218, Las Condes, Santiago de Chile

Teléfono: 56(2) 2080109- Fax: 56 (2) 2203159

Email: eried@netline.cl - eried1@hotmail.com 